\title{
Immunopharmacology Research Including Vaccines in India (2012-2017)
}

\author{
ARUNABHA RAY*, KAVITA GULATI, SURESH KUMAR THOKCHOM and NISHANT RAI \\ Department of Pharmacology, Vallabhbhai Patel Chest Institute, University of Delhi, Delhi 110 007, \\ India
}

(Received on 04 September 2017; Accepted on 05 December 2017)

\begin{abstract}
During the last five years, research in basic and applied immunopharmacology including vaccines has seen various developments. The role of synthetic chemical moieties and recombinant products has been extensively evaluated for their immunosuppressive and immunostimulant properties with considerable degrees of success. Further, research using medicinal plant derived natural agents have gathered significant momentum due to their favorable safety profile coupled with validated beneficial therapeutic effects in certain diseases such as rheumatoid arthritis, bronchial asthma and related allergic disorders, iron deficiency anemia, ulcerative colitis, splenic apoptosis etc. The association of life style diseases precipitated by factors like stress with immune responses has also been investigated. Pharmacological and toxicological studies have shown that oxidative stress and its interactions with nitric oxide may play a major role in such pathophysiological states. Several synthetic and natural agents including antioxidants act by neutralizing the byproducts of body's normal metabolic pathways such as reactive oxygen species (ROS) and reactive nitrogen species (RNS). In the past five years, immunopharmacology research on vaccines also contributed significantly in the development of vaccines and identified most conserved and immunogenic antigens of certain diseases such as brucellosis, hepatitis A and B, plague etc. This can lead to potential vaccine candidates in the future by enhancing vaccine effect on delivering of DNA, immunogenic and mucosal immune response. As some of the novel findings of innovative immunopharmacological research are still in developmental stages, these discoveries will help in bringing various upcoming molecules directed against various immune related disorders in future, which are likely to complement conventional therapy intractable diseases. Immunopharmacology and immunotoxicology are thus likely to emerge one of the disease transforming areas of research with a long-standing impact in biology and medicine.
\end{abstract}

Keywords: Immunopharmacology; Immunomodulators; Vaccines; Antioxidants; Natural Products

\section{Introduction}

The immune system is a host defense system which helps to protect the body against a wide variety of pathogens (Froy et al., 2007). It has evolved to distinguish 'self' from 'non-self' and comprises of many biological structures and processes which has relatively fixed architecture of specialized organs, compound of lymphoid tissues, cells and chemicals. Immune system does a remarkable job of defending against disease-causing microorganisms or various macromolecules that is recognized as non-self or antigen. There are really two interconnected systems of immunity which can be divided into innate immunity (or natural) and adaptive (or acquired) immunity (Aagaard-Tillery et al., 2006). Natural or innate immunity refers to nonspecific defense mechanisms against a foreign antigen that come into play immediately within minutes to hours after infection or appearance of antigen in the body. In this type of immune system, specific recognition of antigen is not required (Vollmar, 2005). On the other hand, in adaptive or acquired immune system, the antigen escapes this natural protective mechanism and invades the host, another set of antigen with specific and powerful defense mechanism are activated after exposure to an antigen either from a pathogen or a vaccination due to "memory" of the cells in immunity (Becker, 2006; Zane, 2001). The acquired immunity is further subdivided into humoral and cell mediated immunity which involved the reaction of lymphocytes. Humoral 
immune response is mediated by antibodies (from Blymphocytes) that bind to antigen followed by neutralizing and removing of the toxic by the process of phagocytosis through opsonization. On the other hand, cell mediated immunity involves cytolytic $\mathrm{T}$ lymphocytes that recognize and activate the macrophages or kill the infected cells directly (Parslow, 2001). The system of defending the body mainly involves multiple and functionally different cell types which provide different varieties of defense mechanisms. The result is a sensitive system of checks and balances that provide an immune response which is prompt, appropriate, effective and self-limiting (Becker, 2006; Zane, 2001). Various cell populations involved in the immune response release different soluble growth and activation factors that play a pivotal role in the initiation, propagation, and regulation of immunologic responses.

Immunopharmacology is a branch of pharmacology concerned with the effects of drugs on the immune system, and involves the amalgamation of two distinct scientific disciplines viz. immunology and pharmacology. It studies the effectiveness of drug components on the immune system and helps to estimate the risks to the immune system from exposure to drugs. The science of immunopharmacology aims to manipulate the immune system by modifying the endogenous immune responses to the benefit of the host in the treatment of diseases. The term immunopharmacology was used for the first time to link the function of the nervous system to the immune system. In the following years, the term was frequently used with reference to the radioimmunoassays of drugs and hormones, and the cyclic nucleotide pharmacology of the immune system (Andor Szentivanyi, 1959). However, the specialty has since undergone evolution to expand its domain and now includes all aspects of inflammation pharmacology, immunomodulatory (immunostimulant and immunosuppressive) agents and immunotherapy. Drugs which modify immune responses are generally categorized as immunomodulators, and could be either immunostimulant or immunosuppressants. There are certain drugs which have both the properties depending on which component of immune response they affect. During immunomodulation, there is enhancement of immune reactions which primarily causes stimulation of a non-specific system, i.e. granulocytes, macrophages, complement, certain T-lymphocytes and different effector substances. Immunosuppression, in turn, acts primarily to reduce resistance against infections, stress and may occur on account of emotional, environmental or chemotherapeutic factors. Suppressive and stimulatory agents have emerged as an important therapeutic modality for immunodeficiency diseases, such as chronic viral infections, and cancer. These compounds act through different principles, on specific molecular targets, for major indications, and have a variety of adverse effects (Nelson and Ballow, 2003). For regulating normal immunological functions, both immunostimulation and immunosuppression are required and thus both these agents have their place in therapeutics. Search for better agents with better efficacy and margin of safety is of major area of contemporary research worldwide (Patwardhan et al., 1990).

\section{Research on Immunomodulatory Activity}

Immunobiology is one of the most rapidly emerging areas of medical biotechnology research and holds great promises with regard to the cure and prevention of a wide range of diseases such as the inflammatory diseases of skin, gut, respiratory tract, joints and central organs. In addition infectious diseases are now primarily considered immunological disorders while neoplastic diseases, organ transplantation and several autoimmune diseases may involve immunosuppressive treatment. Immunosuppressants are drugs which inhibit cellular/humoral or both types of immune responses, and they are commonly used in the treatment of organ transplantation and autoimmune diseases (Tripathi, 2013). Some examples of immunosuppressants are glucocorticoids, calcineurin inhibitors, antiproliferative and antimetabolic agents and antibodies. Immunostimulants are the agents which are envisaged to enhance body's resistance against infections; they enhance the basal levels of immune response, and in individuals with impairment of immune response as immunotherapeutic agent. A number of disorders such as immunodeficiency state, autoimmune disease, cancer and viral infection can be treated with immunostimulant drugs. Newer generation of immunosuppressants, called tolerogens have emerged. Tolerance refers to the specific immunological non-reactivity to an antigen resulting from a previous exposure to the same antigen. While the most important form of tolerance is non-reactivity 
to self-antigens, it is possible to induce tolerance to non-self-antigens. When an antigen induces tolerance, it is termed tolerogen. A tolerogenic response is antigen induced specific non-responsiveness.

\section{Studies on Plant Derived Immunomodulators}

Immunomodulation using medicinal plants has attracted considerable attention due to the recent evidences suggesting their capacity to enhance the body's resistance against certain infections and other related diseases. Immunomodulation using plant secondary metabolites (such as phenolics, flavonoids, and terpenoids) can provide a viable alternative or useful adjunct to conventional treatments for a variety of diseases. Immunomodulatory activities achieved by the use of plant based natural medicines have been reported in several research papers. In recent years, there has been growing interest in the field of herbal drug research and search for cost effective and promising immunomodulatory compounds from naturally occurring substances (Balekar et al., 2014). Thus, herbal drug research and search for cost effective as well as safe therapeutic immunomodulatory options from natural products has been a rapidly expanding area of pharmacology research.

Kumar and Sharma, (2015) evaluated the immunoprotective and other related effects of the traditionally used curcumin from Curcuma longa Linn in deltamethrin (DLM)-induced thymic apoptosis and showed altered immune function (Kumar and Sharma, 2015). Though curcumin is used as immune enhancer, but its underlying mechanism of immune-protection remains unknown. In their study, role of curcumin in DLM-induced thymocyte apoptosis and its changes in immune functions were evaluated. A strong binding affinity towards CD4 and CD8 receptors was revealed in silico studies. Thymocyte apoptosis was shown to be induced by DLM through oxidative stress and caspase-dependent pathways. In the curcumin treatment group, there was inhibition of production of DLM-induced reactive oxygen species, replenishment of deleted glutathione and suppression of caspase activity which may thus be responsible for the suppression of downstream cascade of events, i.e. apoptosis, phenotypic changes and altered cytokine release.
In another study, Sudan et al. (2014), the potency of ethnomedicinally valuable species known as Arisaema jacquemontii (Himalayan Cobra Lily) of North-Western Himalayan region using its tubers, leaves and fruits was measured for its immunomodulatory and antioxidant properties (Sudan et al., 2014). Extract from this plant was found to have better chelating capacity on ferrous ions and ferric ion reducing antioxidant power (FRAP) assay. Further, the potency of its immunomodulatory effect was revealed by significant suppressive effect on mitogen induced T-cell and B-cell proliferation as well as remarkable stimulatory effect on humoral response. Thus, their study concluded that the plant had considerable antioxidant and immunomodulating potential which needs to be explored further.

Sea buckthorn (Hippophae rhamnoides) is an important medicinal resource which is found abundantly in the North Western Himalayan regions. Jain et al. (2013) evaluated. The potentiating effects of sea buckthorn and glucomannan leaves extract (water-soluble polysaccharide that is considered a dietary fiber) was evaluated against ochratoxin induced immunosuppression in Japanese quails (Jain et al., 2013). Their investigation revealed of immunomodulatory effects of both glucomannan and sea buckthorn leaf extracts at dietary level, with combined treatment being more effective for the protection of quails against ochratoxin induced immunosuppression.

Ghatak et al. (2012) evaluated the immunomodulatory potential of oryzanol (OZ), a commercially important bioactive phytochemical component isolated from crude rice bran oil (Ghatak et al., 2012). Significant potential for augmenting immune activity by cellular and humoral mediated mechanisms was evoked which was observed by a significant increase in antibody titer values in haemagglutination test; potentiation of the delayed type hypersensitivity reaction induced by sheep red blood cells, and an increase in phagocytic index in the carbon clearance assay. Thus the findings from the study concluded that $\mathrm{OZ}$ possessed potent immunomodulatory effect.

In another study, immunomodulatory activity of a polyphenolic fraction of Cinnamomum zeylanicum L. (PP-CZ) bark was evaluated against infection- 
related conditions using normal and immunecompromised mice (Balekar et al., 2014). The haemagglutinin (HA) antibody titers (primary and secondary) and delayed type hypersensitivity (DTH) response were measured and PP-CZ was shown to have beneficial effects on the immune system. Their findings revealed that the fraction improved humoral (antibody production), cell mediated responses (DTH) and innate (phagocytosis) responses of the immune system, as well as numbers of resident peritoneal macrophages - suggesting a potent immunomodulatory activity of Cinnamomum zeylanicum.

Patil et al. (2013) studied the immunomodulatory properties of $B$. racemosa fruit extract in experimental animals, which has been used medicinally in treatment of diarrhoea, asthma, coughs, jaundice, analgesic and antipyretic (Patil et al., 2013). Animals treated with the $B$. racemosa extract showed significant decrease in DTH response and a higher antibody titer in haemagglutination assay as compared to control group of animals, suggesting a potent immunomodulatory effect of $B$. racemose.

In vivo experiments were conducted in rodents (Pravansha et al., 2012) to find out possible modulation of cell and humoral immune response by leaf extract of Leptadenia reticulata Linn. commonly known as "dodi". The plant is used as stimulant, tonic, immunostimulant and is one of the ingredients in Ayurvedic formulation called as "Chyawanprash". Various immune parameters such as haemagglutinating antibody titer, haematological profile ( $\mathrm{Hb}, \mathrm{WBC}, \mathrm{RBC})$, lipid peroxidation, reduced glutathione, superoxide dismutase, catalase, DTH response, neutrophil adhesion test and carbon clearance assay were performed. The results obtained from the study showed that Leptadenia reticulata possesses potential immunomodulatory and antioxidant activity.

In another study, (Augustine et al., 2014) evaluated the immunomodulatory activity of widely used ethno-medicinal plant Leucas aspera in Swiss albino mice. The plant also possesses powerful anticancer activity in various cancer cell line studies by stimulating macrophage cells which plays a central role in the immune system. Like other immune modulating plant, this plant was also shown to increase the neutrophil adhesion to the nylon fibres, produced a significant increase in the phagocytic index in carbon clearance test and protection against cyclophosphamide-induced immunosuppression. Further, this plant also showed an increase in the circulating serum immunoglobulins in hemagglutination antibody titer test along with an increase in the foot pad edema in DTH reaction test-suggesting an immunomodulatory profile for Leucas aspera.

Further, Wahab et al. (2014) studied the antioxidant and immunomodulatory activity of tropical and subtropical Indian plant called Bombax ceiba (commonly known as Silk Cotton Tree or Semal) in normal and immunocompromised mice models (Wahab et al., 2014). The study assessed various parameters such as hemagglutinating antibody titer, DTH response, hematological profile ( $\mathrm{Hb}, \mathrm{WBC}, \mathrm{RBC})$, lipid per-oxidation, reduced glutathione, superoxide dismutase, catalase and cytokine (IL- 6 and TNF- $\alpha$ ) release and was shown to have potential immunostimulant properties along with antioxidant capacity.

The immunomodulatory potential of a bioactive fraction from the leaves of Phyllostachys bambusoides (bamboo) was carried out by (Kumar et al., 2014), using a panel of in-vivo assays in balb/ c mice. Stimulation of IgM and IgG titer was observed in haemagglutination assay and also potentiation of the DTH reaction. The levels of IFN- $\gamma$, IL-2, and IL4 were also significantly increased in blood. Furthermore, induction of macrophage phagocytosis, and nitric oxide (NO) production against Candida albicans and carbon clearance test were also reported. Flowcytometry revealed the enhancement in CD4 and CD8 cell populations and thus the study concluded that Phyllostachys bambusoides had an effective immunostimulatory effect by eliciting both Th1 and Th2 mediated immune responses.

Another study also showed the augmenting effect of Cicer microphyllum extract (commonly called as drumstick or horse radish tree) on Th1 immune response in normal and chronically stressed swiss albino mice (Kour et al., 2011). Flowcytometric investigations of T-cell subsets (CD3+, CD4+/CD8+), CD80/CD86, CD28, CD69, co-stimulatory molecules and Th1/Th2 cytokines was carried out and it was shown that the plant enhanced the proliferation and differentiation of $\mathrm{T}$ lymphocytes both in normal and 
chronically stressed mice. Further, DTH response (an indicator of CMI), in-vitro lymphocyte proliferation and Th1/Th2 cytokines in isolated peripheral blood mononuclear cells were analyzed by flowcytometry and the results showed enhanced cytokine expressions in Cicer microphyllum treated group of animals. Moreover, a significant suppression of raised corticosterone levels along with significant expression of important secondary signals for the activation of the immune system such as co-stimulatory molecules, CD28, CD69, CD80 and CD86 were observed in the stressed animals. Thus, the study concluded that Cicer microphyllum possessed immune potentiating and immune recuperative properties in normal and chronically stressed mice.

Ranganathan et al. (2013) also evaluated Cucurbita maxima (popularly known as pumpkin) seeds were evaluated for humoral immune responses in rabbits (Ranganathan et al., 2013). Antibody titer and serum immunoglobulin concentration were estimated along with haematological parameters and significant augmentation in the antibody titer and serum immunoglobulin concentrations were revealed. Thus, their results suggested that Cucurbita maxima seeds has the ability to stimulate humoral immune response in rabbits.

In another study by Reddy et al. (2016), coagulin-L, a withanolide isolated from Withania coagulans was studied for protective activity against TLR4 induced oxidative damage and immune response by regulating mitochondria and NOx-derived ROS in human and murine cells, and mouse model (Reddy et al., 2016). This study found that coagulinL has immunomodulatory effect by suppressing TLR 4 induced immune mediators such as cytokines, growth factors (FGF-basic, VEGF), nitric oxide and intracellular superoxide and thus concluded coagulin$\mathrm{L}$ could be of therapeutic importance.

Immunosuppressive drugs exhibit a high degree of inter-individual and intra-individual pharmacokinetic and pharmacodynamic variability at its critical dose. Therefore, in the present medical system, newer immunosuppressants with negligible or lesser side effects are needed. Medicinal plants can serve as safer therapeutic alternative/adjunct and several studies have shown their beneficial therapeutic effects (Gautam et al., 2007). Thus, in a study by Chauhan et al. (2011) evaluated the immunosuppressive activity of a rare annual herb-Agyrolobium roseum which is grown in north-western Himalayan region of the Indian subcontinent (Chauhan et al., 2011). Aqueous fraction (KA-134) of A. roseum was studied for humoral and cell mediated immune responses and found to be suppressive towards antibody titer and delayed type hypersensitivity reaction. Pinitol is the product which is obtained by further fractioned of KA-134 and was found to have significant suppression of CD3, CD19, CD4 and CD8 counts. Suppression of Th1/Th2 cytokines expression in splenocytes was also observed. It was concluded from these findings that pinitol had a potent and non-toxic immunosuppressive activity.

\section{Studies on Stress-induced Immunomodulation}

Stress is defined as any aversive stimulus external or internal which is capable of disrupting the physiological milieu. The immune system is particularly susceptible to emotional, environmental and xenobiotic stressors and complex mechanisms are involved. Free radicals play a major role in health and disease which is well documented. Reactive oxygen species (ROS) and reactive nitrogen species (RNS) are byproducts of body's normal metabolic pathways and are extremely reactive molecules. There is a delicate balance between pro- and anti- oxidant forces in immune regulation during stress. The mechanisms involved is the defensive/protective role of antioxidants which is poorly understood (Wulf, 2002). In a study by Pal et al. (2011), the role of free radicals during restraint stress (RS)-induced immune response changes was evaluated in experimental animals (Pal et al., 2011). Humoral and cell-mediated immune response was significantly suppressed during RS as evidence by reduced anti-SRBC antibody titer, splenic plaque forming cell counts, footpad thickness response, and the levels of IFN- $\gamma$ and IL-4. Oxidative stress markers such as products of lipid peroxidation (malondialdehyde) and established markers like plasma corticosterone were significantly elevated as compared to controls (no stress). However, there was a reduction in the glutathione levels on exposure to RS in association with reduced antioxidant enzyme activity like superoxide dismutase and catalase. This study revealed that pretreatment with the antioxidants such as L-ascorbic acid and $\alpha$-tocopherol significantly attenuated the above immunological and oxidative 
stress markers, suggesting their synergistic effect with the involvement of reactive oxygen species on reversal of RS-induced effects probably.

Melatonin (n-acetyl-5-methoxytryptamine), a hormone secreted by the pineal gland, helps to control circadian rhythm of the body. Role of melatonin in several diseases including neurological and immunological disorders have been shown in many studies and proved to be both potentiating and protective. Pal et al., (2016) evaluated the protective effects of melatonin in RS-induced behavioral and immunological changes in rats (Pal et al., 2016). After $1 \mathrm{hr}$ RS exposure, significant reduction in open-arm entries, time spent on open-arm in elevated plus maze (EPM) were found and MDA levels were significantly increased in brain homogenate. There is suppression of immunological parameters such as anti-SRBC antibody titer, splenic plaque forming cell (PFC) counts, $\%$ change in paw volume and Th1 (IFN- $\gamma$ ) and Th2 (IL-4) cytokine levels, in both humoral and cellmediated immune responses to antigen. It was concluded that pretreatment with melatonin significantly reversed the above stress induced immune changes and thereby suggesting the involvement of free radicals during stress-induced neurobehavioral and immunological changes.

Another study evaluated the modulatory effects of morphine treatment on elevated plus maze test parameters, oxidative stress markers and Hsp70 expression in acute and chronic stress induced neurobehavioral and cellular markers in rats (Joshi et al., 2014). Prooxidant-antioxidant balance was altered during acute and chronic stress caused by neurobehavioral suppression and Hsp 70 expression in brain homogenates was increased in a differential manner. RS induced anxiogenesis was attenuated by morphine showing changes in MDA and GSH levels but further enhanced Hsp 70 expression. Pretreatment with morphine did not alter the neurobehavioral response to chronic RS but reverted the GSH levels and Hsp 70 expression suggesting that morphine differentially influences acute and chronic stress induced changes in anxiety behavior.

In a recent study, the effects of chronic predictable stress (CPS) and chronic unpredictable stress (CUS) were assessed on immunological responses in $\mathrm{KLH}$-sensitized rats and possible involvement of nitric oxide (NO) mediated signaling pathways mediating such responses were examined. Rats were exposed to either CPS or CUS for 14 days and $\mathrm{IgG}$ antibody levels (DTH) response were determined to assess changes in adaptive immunity. To evaluate the role of nitric oxide during such immunomodulation, biochemical estimation of stable metabolite of nitric oxide (NOx) and 3-nitrotyrosine (3-NT, a marker of nitrosative stress) were done in both blood and brain. Chronic stress exposure resulted in suppression of $\operatorname{IgG}$ and DTH response and elevated both NOx and 3-NT levels, with more marked changes seen with CUS. Pretreatment with aminoguanidine (iNOS inhibitor) caused further reduction of adaptive immune responses and attenuated the increased NOx and 3-NT levels in CPS or CUS exposed rats. On the other hand, 7Nitroindazole (nNOS inhibitor) did not significantly affect these estimated parameters. It was inferred that iNOS and to a much lesser extent nNOS could be involved in the NO mediated modulation of adaptive immunity during stressful experiences and predictability of the stressor played a crucial role in such stress-NO interactions (Thakur et al., 2017).

\section{Immunomodulation and Disease}

\section{Studies on Rheumatoid Arthritis}

Rheumatoid arthritis (RA) is a chronic inflammatory autoimmune disease which affects joints and causing physical disability in the elderly. Due to its unknown etiology, there are still no cure or effective measures for the prevention of this disease. Chauhan et al. (2012) explored the underlying mechanism of chlorogenic acid, a phenolic compound isolated from the aqueous fraction of Bidens pilosa on various immunological parameters in experimental model of rheumatoid arthritis. In nature, chlorogenic acid is widely present in human diet and possesses several properties such as antioxidant, anti-carcinogenic and anti-inflammatory (dos Santos et al., 2006). The total (CD3) and differentiated T cells (CD4 and CD8) count and co-stimulatory molecules (CD28, CD80/ 86) were effectively controlled in Bidens pilosa treatment group - thus suggesting the anti-arthritic potency of chlorogenic acid.

\section{Studies on Bronchial Asthma and COPD}

Bronchial asthma is a heterogenous, chronic inflammatory disease characterized by airway 
inflammation, bronchial hyperresponsiveness, reversible airway obstruction and airway tissue remodeling. Various inflammatory and structural cells are involved for synthesizing and releasing certain inflammatory mediators that are responsible for pathophysiology of asthma (Holgate, 2008). In view of the safety profile of the conventional medications, traditional medicines based on medicinal plants (both monoherbal and polyherbal) are being constantly explored in search of viable alternatives/adjuncts for treatment of various respiratory and allergic diseases including asthma (Kale et al., 2010).

Chaudhary et al. (2016) evaluated the effects of Solanum xanthocarpum in experimental model of asthma. Rats were immunized and challenged with ovalbumin aerosol inhalation. Inflammatory markers such as IgE levels, the concentrations of TNF- $\alpha$, IL6 , IL-4 and IFN- $\gamma$ were evaluated both in blood and bronchoalveolar lavage fluid (BALF), and the levels were found to be attenuated after the treatment with S. xanthocarpum. Further, analysis of oxidative stress parameters such as MDA (lipid peroxidation) was found to be reduced, whereas, GSH (antioxidant) levels were increased. The findings of the study showed that $S$. xanthocarpum has anti-inflammatory and immunomodulatory effects, which could be a beneficial candidate for the treatment of bronchial asthma. Clinical trials are being designed to translate the laboratory findings to the clinic.

Guhathakurta et al. (2013) showed the antiinflammatory and immunomodulatory effects of UNIM-352, a polyherbal Unani preparation, in an experimental model of bronchial asthma in rats (Guhathakurta et al., 2013). In delayed type hypersensitivity experiment, polyherbal preparation did not show any significant change in footpad thickness in KLH sensitized and challenged rats. Further, Rai et al. (2015) conducted experimental studies on possible cellular and molecular mechanisms of action of UNIM-352 in ovalbumin immunized and challenged rats (Rai et al., 2015). Therapeutically beneficial effects were demonstrated in the UNIM-352 treated group and were evidenced by markedly reduced number of eosinophil and neutrophil cells in both blood and BAL fluid as compared to control group. TNF- $\alpha$, IL-4, GM-CSF, NF- $\kappa \mathrm{B}$ and histone deacetylase (HDAC) levels were also found to be attenuated in UNIM-352 treated group. Taken together the study showed the ameliorative effects of UNIM-352 in bronchial asthma.

In another study by Singh et al. (2016), the effects of a secondary plant metabolite, chelidonic acid, were evaluated on mass cell degranulation and adaptive immunity in experimental animals (Singh et al., 2016). The results showed immunomodulatory effects of chelidonic acid-predicting it's therapeutic potential in allergic disorders. Chelidonic acid was shown to attenuate mast cell degranulation in ovalbumin immunized and challenged rats when compared with vehicle control group, and these effects were comparable with those of prednisolone. In chelidonic acid treatment group, there was reduction in mortality, serum IgE levels, blood eosinophil counts, as well as histamine release from peritoneal mast cells, in a dose related manner. Furthermore, the suppression of plaque forming cells counts in splenic cells, anti-SRBC antibody titer and serum IgG levels was reported from the study-suggesting the possible therapeutic potential of chelidonic acid in allergic disorders.

Exacerbations of breathlessness due to lower respiratory tract infection is one of the main causes of chronic obstructive pulmonary disease (COPD) and a prevention from such exacerbation by multicomponent sublingual vaccine (Ismigen), derived from the various causative pathogen is shown along with its tolerability by (Nadig et al., 2015). Thus, COPD patients meeting the eligibility received Ismigen once daily for ten days a month for three consecutive months. Different parameters of respiratory symptoms such as exacerbations, pulmonary function tests, number of hospitalizations and adverse reactions were recorded up to one year and was compared with the mean score parameters of the previous year. In patients receiving Ismigen there was a reduction in exacerbations and mean number of hospitalizations without serious adverse events but there was no significant change in the respiratory symptom score and pulmonary function test. Thus, from their study, they concluded that Ismigen could be used as add on therapy in COPD patients in order to reduce the severity and frequency of exacerbations.

\section{Studies on Iron Deficiency Anemia}

Iron deficiency (ID) anemia is the most common type of anemia in the developing world which is mainly 
associated with the lack of nutritional iron. Iron is an essential micronutrient and its significance in the body is well recognized. The most vulnerable to ID are children and woman in the reproductive age group. Iron plays a very important role in the development of normal immune system and its deficiency results in the generation of specific response to infection as well as immune cell proliferation (UNICEF/UNU/ WHO/MI. 1999).

A case control study was conducted to evaluate the effects of iron deficiency anemia (IDA) and its treatment with oral iron supplementation on cellmediated immunity (CMI) and humoral immunity (HMI) in children. Absolute and relative numbers of CD4 and CD8 lymphocyte subgroups were investigated to assess the CMI and were found to have significantly lower levels in the iron deficient children. On the other hand, iron supplementation, significantly improved the CD4 cell counts as well as CD4:CD8 ratios. Serum Immunoglobulin G (IgG), Immunoglobulin A (IgA), immunoglobulin M (IgM) was carried out to access the HMI at baseline and 3 months post oral iron supplementation but no significant differences in the levels were found between the two groups. Taken together, their study concluded that IDA significantly impaired CMI and it was markedly improved by iron supplementation though there was no influence on HMI (Das et al., 2014).

\section{Studies on Ulcerative Colitis}

Chronic inflammatory bowel disease involves chronic inflammation of all or part of digestive tract. Ulcerative colitis and Crohn's disease are two common chronic inflammatory bowel diseases (IBD). Probiotics are the microbial food supplements (bacteria and yeasts) introduced into the body to promote the host health by its immunomodulatory activity (Sartor, 2004).

Kumar et al. (2017) studied the immunomodulatory and therapeutic potency using a new strain of probiotic in ulcerative colitis Bifidobacterium bifidum 231 (BIF 231) in rats (Kumar et al., 2017). Quantification was done by gram staining, scanning by electron microscopy and q-PCR was used to quantify in vitro adhesion assays and immunomodulatory effect of BIF 231 on interleukins (IL-1 $\gamma$ and IL-10) in IEC- 6 cell lines. The study found a better adhesion and immunomodulation by up-regulation of IL-10 levels in IEC- 6 cell lines and down regulation of IL- $1 \gamma$ levels and suggested a beneficial effect of BIF 231 on damaged tissue in induced colitis model.

\section{Studies on Splenic Apoptosis}

The spleen is the largest organ in the lymphatic system which is important for both the storage and purification of red blood cells and responsible for mounting immune responses to antigens in the blood stream. DLM, a powerful immunotoxicant absorbed by humans from various routes (inhalation, dermal, oral etc) and ultimately reaching the systemic circulation thereby suppressing cytokines and altering the immune functions. Studies using active plant derived compounds have been conducted in order to reduce apoptosis by DLM (Abdul-Hamid and Salah, 2013).

Piperine (the active component in Piper longum /Piper nigrum) was considered one of the drugs of choice under immunocompromised conditions from a comparative study on efficacy of piperine and curcumin in DLM induced splenic apoptosis and altered immune functions (Kumar and Sharma, 2015). Apoptotic markers such as oxidative stress and caspase 3 induced by DLM were significantly attenuated by piperine as compared to curcumin. Moreover, other phenotypic and cytokine changes were also markedly enhanced after piperine. From the findings of their study, they concluded that piperine had anti-oxidative, anti-apoptotic and chemo-protective properties under immunocompromised conditions.

\section{Studies on Insecticides}

Sankhala et al. (2012) conducted a study to evaluate the hematological and immunological changes on short term oral administration of acephate $(\mathrm{O}, \mathrm{S}$-Dimethyl acetyl phosphoramidothioate) - a top ten organophosphate insecticide, sold throughout the world and currently registered for use on a variety of field, fruit, and vegetable crops (Sankhala et al, 2012). A sub-acute toxicity study was done with oral administration of acephate in BALB/c mice and hematological (monocytes and granulocytes), biochemical (serum total protein, serum albumin and globulin), and immunological (humoral immune response and the delayed-type hypersensitivity response to sheep red blood cells) were measured 
after 28 days of oral administration. The study concluded that low concentration exposure to acephate in experimental animals resulted in suppression of immune responses.

\section{Immunopharmacological Studies on Vaccines}

During the last five years, various pharmacological researches in the field of vaccines have enriched our knowledge. Nearly 30 million children in the world are not immunized against preventable diseases due to technological complexity and expensive approaches in vaccine production. Certain specific and specialized requirements of packaging, cold chain and mode of delivery can be mentioned which adds to its cost (Tiwari et al., 2009). Hence, vaccination becomes unaffordable to a large portion of population especially in developing countries with poor socioeconomic status. Mammalian cell line based vaccines, which are the current preferred modality in vaccine production, requires large investments and expertise limiting their scale up and thus, global availability. Similar problems are also being faced in the vaccination of farm animals. It is thus important to initiate a new approach in vaccine development/ manufacture for delivering simpler and affordable vaccines to the population at large.

\section{Studies on Vaccines for Enhancing Immune Response}

Adjuvants are required to enhance the effect of modern recombinant vaccines as they are less immunogenic than conventional vaccines. Thus, in a study by Anugraha et al. (2015), in order to study the augment immune response in several diseases, alum was used as a standard adjuvant for protein based vaccines (Anugraha et al., 2015). The adjuvanticity of Poly-l-lactide (PLA) micro particles was used as a single dose immunization for controlling the problems associated with alum i.e., multiple dose at specific time interval. From their study, fruitful results were obtained that could be applied for the treatment of various diseases. Filarial recombinant chimeric multivalent vaccine candidates such as TV (a $39 \mathrm{kDa}$ fusion protein comprising of two filarial key vaccine candidate antigens such as Thioredoxin and venom allergen homologue) and filarial epitope protein (FEP) are absorbed onto PLA by using double emulsion technique. Further, analysis of PLA encapsulated microparticles was done and evaluated for its immune responses in mice as well. The effectiveness of single dose of PLA encapsulated proteins was compared with single dose of alum or protein alone. They were found to have higher antibody titer values by single dose of PLA encapsulated antigen (such as TV and FEP) than single dose alum absorbed TV/FEP and single dose of protein TV/FEP alone. They also found significant values in the cellular proliferation of cytokine response of IL-4 (Th2) and IFN- $\gamma$ (Th1) by PLA TV encapsulated antigens and high levels of IL-5 (Th2) and IFN- $\gamma$ by PLA-FEP. Thus, they have concluded that the overall strong humoral and cellular responses shown by PLA encapsulated antigens compared with single dose of alum adsorbed or protein alone.

In another study, a tetanus toxoid-loaded cationic non-aggregated and unmodified nanoparticles (TTC-NLPs4 and TTNLPs1) were evaluated for their strong humoral and cellular immune responses (Kaur et al., 2016). Potentially high humoral (anti-TT IgG) and cellular (IFN- $\gamma$ ) immune response were observed by in-vivo immunization with non-aggregated TT-CNLPs4 at day 42 as compare to non- aggregated TTNLPs1. Thus, from the findings of their study, they concluded that such non-aggregated cationic lipid nanoparticles may be a potent immune-adjuvant for parenteral delivery of weak antigens.

In an another study by Biswas et al. (2015), measles antigen loaded low molecular weight chitosan (CS) nanoparticles were developed and subsequently coated by using sodium alginate. The size and surface properties of the nanoparticle can be modulated by changing molecular weight of chitosan polymer. Finding was noted from in vitro studies showing that Makoid-Banakar model was best suited when initial burst release followed by extended release. SDSPAGE assay also showed that alginate coating helps in protecting the antigen in acidic medium for a minimum of 2 hours and thus antigens are protected in gastric environment with sustained release kinetics. Cell viability study was also performed using MTT assay and this formulation was given orally to mice followed by analysis of immunological changes using ELISA method. Obtained results showed that measles antigen loaded CS nanoparticles induced strong immune response and significant correlation was observed. A strong induction in immune response both in systemic and mucosal with low cytotoxicity results 
were observed with measles antigen loaded with CS nanoparticles, which showed a positive application for oral vaccine delivery with low molecular weight CS.

Another study was also conducted by Mitra et $a l$. (2015) on the efficacy and safety of Vi-tetanus toxoid conjugated typhoid vaccine $\left(\right.$ PedaTyph $\left.{ }^{\mathrm{TM}}\right)$ in school children of Kolkata (Mitra et al., 2015). In this study, conjugate vaccine was prepared by binding $\mathrm{Vi}$ to tetanus toxoids (Vi-TT), as Vi polysaccharide typhoid vaccines could not be used in children $<2$ years due to poor immunogenicity and $\mathrm{T}$ cell independent properties. The efficacy and safety levels were measured in children of 6 months to 12 years of age who were selected with permission from the school authority. Groups were randomly divided into vaccinated as test group and unvaccinated as control group. Test group was given 2-doses of vaccine at 6 weeks interval of time and control group received vaccine as per national guidelines. Examination of adverse events (AEs) after vaccination was done at 30 minutes, and 1 month along with clinical events observed till 12 months. Culture positive typhoid fever was found in control group but there was none in vaccine treated group during 12 months. The evaluation of antibody titer values for immunogenicity was done. Sereoconversion was found to be maximal at 6 weeks of post vaccination but lesser at the end of 12 months considering 4-fold rise from baseline showing minimal AEs with maximum efficacy of the vaccine in the first year of follow up. Thus they concluded $100 \%$ protection of Vi conjugate typhoid vaccine in children of 6 months to 12 years of age with effective immunogenicity.

Another similar study on therapeutical dimensions of immunomodulation with adjuncts as novel pharmaceutical interventions was conducted on laboratory animals by Bagherwal and Machawal, 2013. The study evaluated the modulation of immune response to DPT (Diphthera, Pertussis, Tetanus) vaccine by non-dialyzable latex (NDL) from Calotropis procera. NDL of Calotropis procera (Family: Asclepiadaceae) was used for assessment of its immunopotentiation action in animals immunized with DPT vaccine. Serological and hematological parameters were used for evaluation of immunostimulation. B. pertussis (BLC-216 strain) was used to immunize animals and there was a significant enhancement of antibody titers with the
NDL treated animal group. There was overall improvement indicating the development of immune health on behalf of degree of sickness, paralysis and death after intracerebral challenge of $B$. pertussis which is shown by reducing morbidity and mortality of experimental animals. Thus, Calotropis procera offered direct therapeutic benefits with its possible immunopotentiating applications.

A study was conducted for identification of most potential and immunogenic antigen of Streptococcus pneumoniae by reverse vaccinology, which can be a potential vaccine candidate in the future (Talukdar $e t$ al., 2014). BLASTn (nucleotide basic local alignment search) is a tool used most widely to identify the most potential antigen and also to compare the similarity among two or more nucleotide or protein sequences. PSORTb 3.0.2 is a computational tool which can predict a protein's subcellular localization which is of great help in genome analysis. In their study, immunogenicity testing was used for the prediction of B cell epitope, and BLASTp was performed for verifying the extent of similarity to human proteome to exclude the possibility of autoimmunity. At each step, the proteins that failed to comply were filtered. Using this, several pneumococcal proteins were screened in which pavB and pullulanase were reported to be most promising protein candidate.

In an another study, using lysinylated amphiphiles with manose-mimicking head-group, a long-lasting dendritic cell DNA vaccination system was proposed by (Srinivas et al., 2012). As dendritic cells (DCs) pulsed/traduced with tumor-associated cells or viral antigens, they were used for fighting cancer and infectious diseases and thus there was a possibility of developing a potential dendritic cells-based genetic immunization (DNA vaccination) system for providing truly long-lasting protective immunity. Applying this concept, a plasmid DNA encoding melanoma tumor associated antigen and liposomes of two lysinylated cationic amphiphiles with mannose-mimicking quinic and shikimic acid head-groups was used. This was then immunized with an autologous DCs pretransfected with electrostatic complexes (lipoplexes). They reported that 300 days post tumor challenge yielded a protective immunity with significant memory response of more than six months after the second tumor challenged in the immunize group. Thus, they have shown the possibility of inducing long-lasting 
immune response in DC-based genetic immunization with non-viral ex vivo DC-transfection system.

\section{Vaccine Research for Enhancing Delivery of DNA Vaccine}

Lactococcus lactis apart from widely being used in food industry, has also been commonly used as an antigen as well as vehicle for DNA delivery of newly constructed immunostimulatory DNA vaccine reporter plasmid such as pPERDBY. In order to enhance the DNA delivery efficiency of L. lactis, Yagnik et al. (2017), investigated dual recombinant L. lactis expressing Internalin A of Listeria monocytogenes and harbouring pPERDBY (LL InlA+ pPERDBY) (Yagnik et al., 2017). The results were found to be significantly effective when compared to previously develop non-invasive r- L. lactis::pPERDBY and from this finding it was suggested to have potential application of invasive strain of L. lactis in delivery of DNA/RNA and antigens.

\section{Vaccine Research on Immunogenic and Mucosal Immune Response}

Expression of outer membrane protein A (OmpA) of Shigella dysenteriae type-1 (SD-1) by Lactococcus lactis when administered either orally or intranasally was evaluated in a study by Yagnik et al., 2017. OmpA of SD-1 was cloned and expressed first in Escherichia coli (E. coli) and then in L. lactis. OmpA was purified after it was cloned and expressed in E. coli and then in L. lactis and subcutaneously administered in BALB/c mice. Immunogenicity of OmpA was confirmed by detection of OmpA specific IgG antibodies and IgA by enzyme linked immunosorbent assay (ELISA) and compared for both the routes. The study concluded that recombinant $L$. lactis expressing OmpA of SD-1 was found to be immunogenic and also oral administration of recombinant - L. lactis elicited higher systemic and mucosal immune response when compared to the nasal route.

\section{Vaccines and their Effect on Diseases}

\section{Studies on Brucellosis - A Worldwide Zoonotic Disease}

Jain et al. (2014) worked on a worldwide zoonotic disease - brucellosis, where for which no vaccine is available for the use in humans and further there is also limitation for the existing animal vaccines. In their study, they have generated and evaluated the potency of ribosomal protein L9 based DNA vaccine (pVaxL9) in mouse model with intramuscular immunization (Jain et al., 2014). Generation of anti-L9 IgG antibody response of both $\operatorname{IgG} 1$ and $\mathrm{IgG} 2 \mathrm{a}$ isotypes was reported with the pVaxL9 immunized group and thus higher antibody responses were observed. The mice groups immunized with pVaxL9 priming and recombinant L9boosting (PB) were found to have higher level of antibody response and where pDNA immunization was carried out by in vivo electroporation. Proliferation of splenocytes and release of certain cytokines such as IFN- $\gamma$, TNF- $\alpha$, IL-2 was reported. By flowcytometric analysis, release of IFN- $\gamma$ both by CD4+ and CD8+ T cells was revealed and it was thus concluded that L9 based DNA vaccine was immunogenic conferring significant protection against $B$. abortus.

\section{Studies on Hepatitis A}

A mass immunization with a single dose of live attenuated HAV (IV phase study) was done by Mitra et al. (2015) on a worldwide disease - viral hepatitis A with a follow up of 5 years and shown that it significantly provided long-term immunogenicity in healthy Indian children. The study was carried out at four centers of India - Kolkata, Delhi, Mumbai and Chennai. For this, subjects who were having antibody titer $<20 \mathrm{mIU} / \mathrm{ml}$ at baseline were recruited for long term immunogenicity analysis and a total of 503 participants were enrolled, out of which 349 subjects were having a baseline seronegative with anti-HAV antibody titer $<20 \mathrm{mIU} / \mathrm{mL}$. Follow up schedules were made on 6 weeks, 6 months, 12 months, 24 months, 36 months, 48 months and 60 months during this 5 years post vaccination considering seroprotection levels of anti-HAV antibody. At the end of 5 years who came for follow-up had a protective antibody titer $>20 \mathrm{mIU} / \mathrm{mL}$ with increased geometric mean concentration over the years. Taken together, the study concluded a well-tolerated and long term immunogenic effect of single dose of live attenuated hepatitis A vaccine in healthy Indian children.

\section{Studies on Hepatitis B}

A novel sarsasapogenin glycoside (immunoside) from Asparagus racemosus was investigated (Sidiq et al., 
2011) for its protective immune response against hepatitis B surface antigen (HBsAg). The response of immunoside adjuvanted with $\mathrm{HBsAg}$ was compared with alum adjuvanted with $\mathrm{HBsAg}$. Antibody titre (IgG, IgG1/IgG2a), cell proliferation, cytokines (IL-2, IFN$\gamma$ and IL-4), and lymphocyte sub-populations (CD4/ CD8, CD3 and CD19) were measured in mice sera and their results suggested that immunoside potentially enhanced anti-HBsAg immune response via augmenting Th1/Th2 response in a dose dependent manner with its safety evaluation report.

In another similar study by Sidiq et al. (2013), two novel lipidated tripeptide lysine derivatives (KKSM and KKSMB) were evaluated for their potential effect against $\mathrm{HBsAg}$ in various in vitro and animal-derived models of humoral and cellmediated immune events (Sidiq et al., 2013). More stimulation of anti-HBsAg IgG, neutralizing antibody (IgG1 and IgG2a) titres values and T-lymphocyte subsets (CD4/CD8) along with other soluble mediators of Th1 and Th2 in spleen, were found in mice sera when compared with alum adjuvanted with HBsAg. Moreover, enhancement of cell populations (CD4, CD8, CD3 and CD19) and other CD4/CD8 derived cytokine (IL-2, IL-4, IFN- $\gamma$ and TNF- $\alpha$ ) were reported from these lapidated tripeptides in the whole blood of treated group. There was also significant release of IL-12, IFN- $\gamma$ and nitrite content in macrophage supernatant along with enhancement in the population of CD80 and CD86 in spleen-derived macrophages without showing any hemolytic effect on rabbit RBC. Taking these into account, they suggested both these molecules were the potential enhancers of anti-HBsAg immune response via augmenting Th1/Th2.

Severe morbidity and fatality rate due to overlying acute or chronic hepatitis B (HBV) infection is more likely with patients with chronic liver disease (CLD). Though the hepatitis $\mathrm{B}$ vaccines are shown to be safe and effective in patients with CLD, there is still lack of data in liver cirrhosis patients and thus assessment on safety, efficacy and immunogenicity of HBV was done by Roni et al. (2013). In their study, CLD patients who were negative to hepatitis B surface antigen and antibody to hepatitis B core antigen were recruited. They were given three doses of hepatitis B vaccine at 0,3 and 60 days. Anti-HBs antibody was measured after 120 days and found to have less antibody response in the alcoholic chronic liver disease as compare to those who have other cause of chronic liver disease like cryptogenic and hepatitis $\mathrm{C}$ virus infection. The study concluded that low hepatitis B antibody titers were seen with liver cirrhosis patient when compared to general population. With the progress of age and liver disease, these patients had low responses from the hepatitis B vaccination.

\section{Studies on Plague}

Modulation of immune and protective potential of F1 and LcrV antigens of Yersinia pestis by HSP70 Domain II of Mycobacterium tuberculosis in a mouse model was evaluated in a study by Batra $e t$ al. (2014). Y. pestis causes deadly dangerous disease known as plague and no ideal vaccine exists to control it. In their study, they cloned, expressed and purified recombinant $\mathrm{F} 1, \mathrm{LcrV}$ antigens of $Y$. pestis and heat shock protein70 (HSP70) domain II of $M$. tuberculosis in E. coli. Humoral and cell mediated immune responses were evaluated. Immunized animals were challenged with 100 LD50 of $Y$. pestis via intra-peritoneal route. There was generation of significantly high titres of anti-F1 and anti-LcrV IgG antibodies. Significant difference in cytokines (IL-2, IFN- $\gamma$ and TNF- $\alpha$ ) expression and increased percentages of $\mathrm{CD} 4+$ and $\mathrm{CD} 8+\mathrm{T}$ cells producing IFN-ã in spleen of vaccinated animals were observed in comparison to control group. Histopathological studies also revealed effective protection of lung, liver, kidney and spleen of immunized animals and from these findings it was suggested that HSP70(II) of $M$. tuberculosis could be a potent immunomodulator for $\mathrm{F} 1$ and LcrV containing vaccine candidates against plague.

\section{Studies on Lymphatic Filariasis}

Misra et al. (2016) targeted for developing a novel alternative drug against the vector borne neglected tropical disease, known as lymphatic filariasis, caused by human pathogenic nematode Brugia malayi was targeted for developing a novel alternative drug against it. or this, they used UDP-Galactopyranose mutases (UGM) was used as a promising drug target which is involved in the conversion of UDP-Galactopyranose (substrate) to UDP-Galactofuranose (product) and was found to be effective against B. malayi suggesting that $B$. malayi UDP-Galactopyranose mutase could 
be a potential anti-filarial therapeutic drug target against lymphatic filariasis.

\section{Conclusions}

Novel developments in immunopharmacology research help in bringing to the fore various potential and upcoming molecules directed against different immune related disorders. Most of these new findings are still in early developmental stages. However, the progress made so far in explaining the underlying mechanism of the new molecules towards immune

\section{References}

Aagaard-Tillery K M, Silver R and Dalton J (2006) Immunology of normal pregnancy Semin Fetal Neonatal Med 11279 295

Abdul-Hamid M and Salah M (2013) Lycopene reduces deltamethrin effects induced thyroid toxicity and DNA damage in albino rats J Basic Appl Zoo 66 155-163

Anugraha G, Madhumathi J, Jeya Prita P J and Kaliraj P (2015) Biodegradable poly-1-lactide based microparticles as controlled release delivery system for filarial vaccine candidate antigens Eur J Pharmacol 747 174-180

Augustine B B, Dash S, Lahkar M, Amara V R, Samudrala P K and Thomas J M (2014) Evaluation of immunomodulatory activity of ethyl acetate extract of Leucas aspera in Swiss albino mice Int J Green Pharm 8 84-89

Bagherwal P and Machawal L (2013) Immune response modulation to DPT (diphtheria, pertussis, tetanus) vaccine by ndl from Calotropis procera Int J Pharm Bio Sci 4 852-859

Balekar N, Bodhankar S, Mohan V and Thakurdesai P A (2014) Modulatory activity of a polyphenolic fraction of Cinnamomum zeylanicum L. bark on multiple arms of immunity in normal and immunocompromised mice J App Pharm Sci 4 114-122

Batra L, Verma S K, Nagar D P, Saxena N, Pathak P, Pant S C and Tuteja U (2014) HSP70 domain II of Mycobacterium tuberculosis modulates immune response and protective potential of $\mathrm{F} 1$ and LcrV antigens of Yersinia pestis in a mouse model PLoS Negl Trop Dis 8 e3322

Becker K (2006) Innate and adaptive immune responses in CNS disease Clin Neurosci Res 6 227-236

Biswas S, Chattopadhyay M, Sen K K and Saha M K (2015) Development and characterisation of alginate coated low related disorders is a definite advancement. Most of the research on immunopharmacology is designed to complement conventional therapy for advanced disease. Further research and development in immunopharmacology will bring novel molecules and newer targets to the forefront. The domain of immunopharmacology research has now extended to both communicable and non-communicable disorders and developing effective strategies for prevention and cure of various intractable disorders including cancer, HIV-AIDS and autoimmune disorders may not be such a distant dream after all.

molecularweight chitosan nanoparticles as new carriers for oral vaccine delivery in mice Carbohydr Polym 121 403-410

Chaudhary S, Gulati K, Rai N and Ray A (2016) Evaluation of anti-inflammatory and immunomodulatory effects of aqueous extract of solanum xanthocarpum in experimental models of bronchial asthma EC Pharmacol \& Toxicol 2 241-250

Chauhan P S, Gupta K K and Bani S (2011) The immunosuppressive effects of Agyrolobium roseum and pinitol in experimental animals Int Immunopharmacol 11 286-291

Chauhan P S, Satti N K, Sharma P, Sharma V K, Suri K A and Bani S (2012) Differential effects of chlorogenic acid on various immunological parameters relevant to rheumatoid arthritis Phytother Res 26 1156-1165

Chidambaram K, Albert J, Karpagam K, Sivasubramanian and Noohu (2011) Antipyretic activity of crateva magna bark on tab-vaccine induced pyrexia Int J Pharm Sci Res 2 856859

Das I, Saha K, Mukhopadhyay D, Roy S, Raychaudhuri G, Chatterjee M and Mitra P K (2014) Impact of iron deficiency anemia on cell-mediated and humoral immunity in children: A case control study J Nat Sci Biol Med 5 158163

dos Santos M D, Almeida M C, Lopes N P and de Souza G E (2006) Evaluation of the anti-inflammatory, analgesic and antipyretic activities of the natural polyphenol chlorogenic acid Biol Pharm Bull 29 2236-2240

Froy O, Hananel A, Chapnik N and Madar Z (2007) Differential effect of insulin treatment on decreased levels of betadefensins and Toll-like receptors in diabetic rats $\mathrm{Mol}$ Immunol 44 796-802 
Gautam R, Saklani A and Jachak S M (2007) Indian medicinal plants as a source of antimycobacterial agents $J$ Ethnopharmacol 110 200-234

Ghatak S B and Panchal S J (2012) Investigation of the immunomodulatory potential of oryzanol isolated from crude rice bran oil in experimental animal models Phytother Res 26 1701-1708

Guhathakurta S, Gulati K, Rai N, Banerji B D, Jamil S S and Ray A (2013) An experimental study to evaluate the antiinflammatory and immunmodulatory effects of UNIM352, a polyherbal preparation for bronchial asthma Medicinal Plant Research 3 3-12

Holgate S T (2008) Pathogenesis of asthma Clin Exp Allergy 38 872-897

Jain A, Varshneya C and Bhardwaj P (2013) ochratoxin induced immunosuppression and its protection by seabuckthorn (Hippophae Rhamnoides) and glucomannan in Japanese Quails (Coturnix Coturnix Japonica) Indian Vet J 90 44-46

Jain S, Afley P, Dohre S K, Saxena N and Kumar S (2014) Evaluation of immunogenicity and protective efficacy of a plasmid DNA vaccine encoding ribosomal protein L9 of Brucella abortus in BALB/c mice Vaccine 32 4537-4542

Joshi JC, Ray A and Gulati K (2014) Differential modulatory effects of morphine on acute and chronic stress induced neurobehavioral and cellular markers in rats Eur $J$ Pharmacol 729 17-21

Kale R N, Patil R N and Patil R Y (2010) Asthma and herbal drugs Int J Pharm Sci Res $137-42$

Kaur A, Jyoti K, Rai S, Sidhu R, Pandey R S, Jain U K, Katyal A and Madan J (2016) Tetanus toxoid-loaded cationic nonaggregated nanostructured lipid particles triggered strong humoral and cellular immune responses J Microencapsul 33 263-273

Kour K, Sangwan P L, Khan I, Koul S, Sharma S N, Kitchlu S and Bani S (2011) Alcoholic extract of Cicer microphyllum augments Th1immune response in normal and chronically stressed Swiss albino mice J Pharm Pharmacol 63267 277

Kumar A and Sharma N (2015) Comparative efficacy of piperine and curcumin in deltamethrin induced splenic apoptosis and altered immune functions Pestic Biochem Physiol 119 $16-27$

Kumar A, Sasmal D, Jadav S S and Sharma N (2015) Mechanism of immunoprotective effects of curcumin in DLM-induced thymic apoptosis and altered immune function: an in silico and in vitro study Immunopharmacol Immunotoxicol $\mathbf{3 7}$ 488-498

Kumar S, Sharma G, Sidiq T, Khajuria A, Jain M, Bhagwat D and Dhar K L (2014) Immunomodulatory potential of a bioactive fraction from the leaves of phyllostachys bambusoides (bamboo) in balb/c mice EXCLI J 17 137150

Misra S, Valicherla G R, Mohd Shahab, Gupta J, Gayen J R and Misra-Bhattacharya S (2016) UDP-Galactopyranose mutase, a potential drug target against human pathogenic nematode Brugia malayi Pathog Dis $\mathbf{7 4}$ pii: ftw072

Mitra M, Shah N, Faridi M, Ghosh A, Sankaranarayanan V S, Aggarwal A, Chatterjee S, Bhattacharyya N, Kadhe G, Vishnoi G and Mane A (2015) Long term follow-up study to evaluate immunogenicity and safety of a single dose of live attenuated hepatitis A vaccine in children Hum Vaccin Immunother 11 1147-1152

Mitra M, Shah N, Ghosh A, Chatterjee S, Kaur I, Bhattacharya $\mathrm{N}$ and Basu S (2016) Efficacy and safety of vitetanus toxoid conjugated typhoid vaccine (PedaTyph ${ }^{\mathrm{TM}}$ ) in Indian children: School based cluster randomized study Hum Vaccin Immunother 12 939-945

Nadig PD, Haran A and Shetty M (2015) Clinical evaluation of a sub-lingual vaccine for prevention of exacerbations in COPD Asian J Med Sci 6 51-54

Nelson R P Jr and Ballow M (2003) Immunomodulation and immunotherapy: drugs, cytokines, cytokine receptors, and antibodies J Allergy Clin Immunol 111 720-743

Pal R, Gulati K, Banerjee B and Ray A (2011) Pharmacological and biochemical studies on the role of free radicals during stress-induced immunomodulation in rats Int Immunopharmacol 11 1680-1684

Pal R, Gulati K, Banerjee B D and Ray A (2016) Pharmacological and biochemical studies on the protective effects of melatonin during stress-induced behavioral and immunological changes in relation to oxidative stress in rats Can J Physiol Pharmacol 94 296-301

Parslow T G (2001) Medical Immunology 10th Edn.,Lange Medical Books/McGraw-Hill Medical Publishing Division, New York, pp:814

Patil P R, Patil M R, Mane A and Patil S (2013) Immunomodulatory effects of fruits of Barringtonia racemosa Linn Int J Basic Clin PharmacoL 2 216-219

Patwardhan B, Kalbag D, Patki P S and Nagsampagi B A (1990) Search of immunomodulatory agents: a review Indian Drugs 28 348-358

Pravansha S, Thippeswamy B S and Veerapur V P (2012) Immunomodulatory and antioxidant effect of Leptadenia reticulata leaf extract in rodents: possible modulation of cell and humoral immune response Immunopharmacol Immunotoxicol 34 1010-1019 
Rai N, Ray A, Jamil S S and Gulati K (2015) Cellular and molecular mechanisms of action of polyherbal preparation UNIM-352, in experimental models of bronchial asthma Indian J Exp Biol 53 625-631

Ranganathan V, Selvasubramanian S and Vasanthakumar S (2013) Estimation of humoral immune response in rabbits fed with Cucurbita maxima seeds Vet World 6 396-399

Reddy S S, Chauhan P, Maurya P, Saini D, Yadav P P and Barthwal M K (2016) Coagulin-L ameliorates TLR4 induced oxidative damage and immune response by regulating mitochondria and NOX-derived ROS Toxicol Appl Pharmacol 309 87-100

Roni D A, Pathapati R M, Kumar A S, Nihal L, Sridhar K and Tumkur Rajashekar S (2013) Safety and efficacy of hepatitis B vaccination in cirrhosis of liver $A d v$ Virol Article ID 196704

Sankhala LN, Tripathi S M, Bhavsar S K, Thaker A M and Sharma P (2012) Hematological and immunological changes due to short-term oral administration of acephate Toxicol Int 19 162-166

Sartor R B (2004) Therapeutic manipulation of the enteric microflora in inflammatory bowel diseases: antibiotics, probiotics, and prebiotics Gastroenterology 126 16201633

Satish Kumar C S, Kondal Reddy K, Boobalan G, Gopala Reddy A, Sudha Rani Chowdhary C H, Vinoth A, Jayakanth K and Srinivasa Rao G (2017) Immunomodulatory effects of Bifidobacterium bifidum 231 on trinitrobenzenesulfonic acid-induced ulcerative colitis in rats Res Vet Sci 110 40-46

Sidiq T, Khajuria A, Shafi S, Ismail T, Sampath Kumar H, Kannappa Srinivas V, Krishna E and Kamal Johri R (2013) Efficacy evaluation of two synthetic lysine lipidated tripeptides as vaccine adjuvants against HBsAg Eur $J$ Pharmacol 705 68-78

Sidiq T, Khajuria A, Suden P, Singh S, Satti N K, Suri K A, Srinivas V K, Krishna E and Johri R K (2011) A novel sarsasapogenin glycoside from Asparagus racemosus elicits protective immune responses against $\mathrm{HBsAg}$ Immunol Lett 135 129-135

Singh D K, Gulati K and Ray A (2016) Effects of chelidonic acid, a secondary plant metabolite, on mast cell degranulation and adaptive immunity in rats Int Immunopharmacol $\mathbf{4 0}$ 229-234

Srinivas R, Garu A, Moku G, Agawane S B and Chaudhuri A (2012) A long-lasting dendritic cell DNA vaccination system using lysinylated amphiphiles with mannose- mimicking head-groups Biomaterials 33 6220-6229

Sudan R, Bhagat M, Gupta S, Singh J and Koul A (2014) Iron (FeII) Chelation, Ferric Reducing Antioxidant Power, and Immune Modulating Potential of Arisaema jacquemontii (Himalayan Cobra Lily) Biomed Res Int Article ID 179865

Talukdar S, Zutshi S, Prashanth K S, Saikia K K and Kumar P (2014) Identification of potential vaccine candidates against streptococcus pneumoniae by reverse vaccinology approach Appl Biochem Biotechnol 172 3026-3041

Thakur T, Gulati K, Rai N and Ray A(2017) Experimental studies on possible regulatory role of nitric oxide on the differential effects of chronic predictable and unpredictable stress on adaptive immune responses Int Immunopharmacol 50 236242

Tiwari S, Verma P C, Singh P K and Tuli R (2009) Plants as bioreactors for the production of vaccine antigens Biotechnol Adv 27 449-467

Tripathi K D (2013) Essentials of medical pharmacology, $7^{\text {th }}$ edn., Jaypee Brothers Medical Publishers (P) Ltd, New Delhi, pp: 878

UNICEF/UNU/WHO/MI (1999) Preventing iron deficiency in women and children. Technical Consensus on key issues. Boston, MA: International Nutrition Foundation; pp: 160

Vollmar A M (2005) The role of atrial natriuretic peptide in the immune system Peptides 26 1086-1094

Wahab S, Hussain S, Farooqui A H A, Ahmad M P, Hussain M S, Rizvi A, Ahmad M F and Ansari N H (2014) In vivo Antioxidant and immunomodulatory Activity of Bombax ceiba Bark - Focusing on its Invigorating Effects Am J of Adv Drug Deliv 2 1-13

Wulf D (2002) Free radicals in the physiological control of cell function Physiol Rev 82 47-95

Yagnik B, Sharma D, Padh H and Desai P (2017) Dual recombinant Lactococcus lactis for enhanced delivery of DNA vaccine reporter plasmid pPERDBY Microbiol Immunol 61 123129

Yagnik B, Sharma D, Padh H and Desai P (2017) Immunization with $\mathrm{r}$-Lactococcus lactis expressing Outer membrane protein A (OmpA) of Shigella dysenteriae type-1: Evaluation of oral and intranasal route of administration $J$ Appl Microbiol 122 493-505

Zane H D (2001) Immunology: Theoretical and Practical Concepts in Laboratory Medicine. $1^{\text {st }}$ Edn., W.B. Saunders Company, United States, pp: 366. 